\title{
Immune-mediated neurological syndrome in SARS-CoV-2 infection: a review of literature on autoimmune encephalitis in COVID-19
}

\author{
Alvin Oliver Payus ${ }^{1} \mathbb{D} \cdot$ Mohammad Saffree Jeffree ${ }^{1} \cdot$ May Honey $\mathrm{Ohn}^{2} \cdot$ Hui Jan $\operatorname{Tan}^{3} \cdot$ Azliza Ibrahim $^{4}$. \\ Yuen Kang $\mathrm{Chia}^{5} \cdot$ Azman Ali Raymond $^{6}$
}

Received: 6 October 2021 / Accepted: 23 November 2021 / Published online: 1 December 2021

(c) Fondazione Società Italiana di Neurologia 2021

\begin{abstract}
Introduction The novel Coronavirus Disease 2019 (COVID-19) is an infection caused by the Severe Acute Respiratory Syndrome Coronavirus 2 (SARS-CoV-2) which has been spreading rapidly amongst humans and causing a global pandemic. The notorious infection has shown to cause a wide spectrum of neurological syndrome, including autoimmune encephalitis. Objective Here, we systematically review the literature on autoimmune encephalitis that developed in the background of SARS-CoV-2 infections and also the possible pathophysiological mechanisms of auto-immune mediated damage to the nervous system.

Methodology An exhaustive search was made in Medline/PubMed, Embase, Scopus and other medical databases, and 28 relevant published articles were selected according to the strict inclusion criteria.

Results Autoimmune encephalitis can occur via three possible proposed pathophysiological mechanism and can manifest during or after the acute infection period. It is more common in adult but can also occur in the paediatric patients. There were various spectra of autoantibody panels reported including antineuronal antibody, anti-gangliosides antibody and onconeural antibody. Majority of the patients responded well to the immunomodulating therapy and achieved good recovery.

Conclusion In conclusion, SARSCoV-2 infection can induce various spectrum of autoimmune encephalitis. It is a major concern since there is very limited long-term study on the topic. Hence, this review aims to elucidate on the potential longterm complication of SARS-CoV-2 infection and hopefully to improve the management and prognosis of COVID-19.
\end{abstract}

Keywords COVID-19 $\cdot$ SARS-CoV-2 $\cdot$ Pandemic $\cdot$ Neurology $\cdot$ Autoimmune $\cdot$ Encephalitis

Alvin Oliver Payus

dralvinpayus@ums.edu.my

Mohammad Saffree Jeffree

saffree@ums.edu.my

May Honey Ohn

mayhoney.ohn@gmail.com

Hui Jan Tan

tanhuijan@gmail.com

Azliza Ibrahim

maizarah_84@yahoo.com

Yuen Kang Chia

cyk1118@gmail.com

Azman Ali Raymond

drraymondazmanali@gmail.com

1 Faculty of Medicine and Health Science, Universiti Malaysia Sabah (UMS), Jalan UMS, 88400 Kota Kinabalu, Sabah, Malaysia

2 Cardiology Department, University Lewisham Hospital, High Street, London SE13 6LH, UK

3 Department of Internal Medicine, Universiti Kebangsaan Malaysia Medical Centre (UKMMC), Jalan Yaacob Latif, 56000 Cheras, Kuala Lumpur, Malaysia

4 Department of Neurology, Hospital Pengajar Universiti Putra Malaysia, Persiaran Mardi - UPM, 43400 Serdang, Selangor, Malaysia

5 Neurology Unit, Department of Medicine, Queen Elizabeth Hospital, 13a Jalan Penampang, 88200 Kota Kinabalu, Sabah, Malaysia

6 Department of Medicine, Faculty of Medicine, Universiti Teknologi MARA, Jalan Hospital, 47000, Sungai Buloh, Selangor, Malaysia 


\section{Introduction}

The Severe Acute Respiratory Syndrome Coronavirus 2 (SARS-CoV-2) is a newly emerged zoonotic virus that causes the Coronavirus Disease 2019 (COVID-19) [1]. It was first reported in Wuhan, Hubei Province, China on 29 December 2019, where four cases of an acute respiratory syndrome of unknown aetiology were linked to a local Huanan South China Seafood Market and have been causing a global catastrophic since then [2].

SARS-CoV-2 is an enveloped, single-stranded positivesense RNA genome virus, which has a spherical or oval shape and an average size of $100 \mathrm{~nm}$ in diameter. There are large club-shaped spikes of glycoprotein membrane on the viral surface, which makes the viral particles appear like a typical crown-like shape in an electron microscope [3]. Taxonomically, the SARS-CoV-2 is part of the SARSrelated coronaviruses species that belong to the subgenus Sarbecovirus, along with the other subgenuses namely Embecovirus, Hibecovirus, Merbecovirus and Nobecovirus which are part of the genus Beta-coronavirus [4]. These viruses are more common in animals worldwide. However, there are a few types, such as HCoV-HKU1, HCoV-OC43, HCoV-229E and HCoVNL63 that are known to affect the humans and cause a mild respiratory illness $[5,6]$. And there are some that have caused a large-scale epidemic before in the last two decades, such as the case of the Severe Acute Respiratory Syndrome (SARS-CoV) in the year 2002 to 2003, where it has infected more than 8400 individuals with the mortality rate of $11 \%$ across 26 countries around the world [7,8], and then the MiddleEast Respiratory Syndrome (MERS-CoV) in the year 2012 to 2013 which has affected about 2500 people and caused the death of 850 people [9]. SARS-CoV-2 is the first coronavirus to have caused a global range pandemic.

The SARS-CoV-2 infection results in a syndrome of various systemic and respiratory symptoms such as dry cough, breathing difficulty, fever and fatigue that sometimes can be critical causing severe pneumonia and cardiorespiratory failure that require specialized management in intensive care units $[10,11]$. In addition to that, it is well documented that SARS-CoV-2 infection can affect the nervous system causing a wide range of neurological symptoms [11]. Autoimmune encephalitis, which is an immune-mediated inflammatory condition involving the brain tissue, is one of the syndromes that have been reported to occur in the background of SARS-CoV-2 infection. It has been postulated that the autoinflammatory condition was triggered in response to the viral infection in addition to any other environmental confounding factors in a genetically susceptible patient [12]. This condition will have a long-term implication on the patient who recovered from the SARS-CoV-2 infection. Therefore, in the light of the possibility of autoimmune disorders linked to SARS-CoV-2 infection, this review aims to summarize all the published reporting of autoimmune encephalitis that occurs in COVID-19 patients and hopefully provide useful information for the diagnosis and management of the condition.

\section{Methods}

An exhaustive search of scientific publications which consist of a case series and reports was conducted using the following online databases: Medline/PubMed, Embase, Scopus, Web of Science, as well as Google scholar. The search MeSH term keywords used were: ["Encephalitis", OR "autoimmune encephalitis", OR "limbic encephalitis", OR "NMDA-encephalitis"\} AND \{ "SARS-CoV-2", OR "COVID-19", OR "coronavirus" \}. All relevant articles were analysed for the possible autoimmune encephalitis related to COVID-19. These articles were published from 1 January 2020 to 31 July 2021 . There were 45 articles that were organized according to the search words. The reference list of these articles was also searched and analysed for additional reporting related to autoimmune encephalitis in COVID-19 disease which identifies additional 15 articles. A flow chart of the search process is shown in Fig. 1. These articles were then carefully filtered for their relevance based on selection criteria. Amongst the selection criteria that were considered: diagnosed case of COVID19 , confirmed case of autoimmune encephalitis either by the presence of the serum or cerebrospinal fluid autoantibodies or the typical radiological findings and have a clear description of the clinical cases. The occurrence of autoimmune encephalitis in COVID-19 is explored, and a brief review is then performed.

\section{Results}

We reviewed more than 60 scientific papers on autoimmune encephalitis that occurs in confirmed cases of SARS-CoV-2 infection that were published since 1 January 2020 to 31 July 2021 which include limited case series and case reports. We focused on the details on how the diagnosis of autoimmune encephalitis was made based on the serology and radiology investigations done, treatment given and the prognosis of each case. After considering the selection criteria, 26 case reports and 2 limited case series are included in the study. A brief summary of the selected articles is briefly described in Table 1 . 
Fig. 1 Flow chart of the literature search process according to PRISMA guidelines

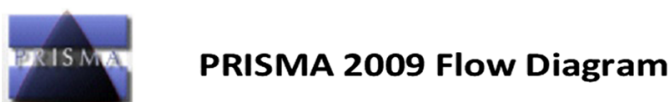

PRISMA 2009 Flow Diagram

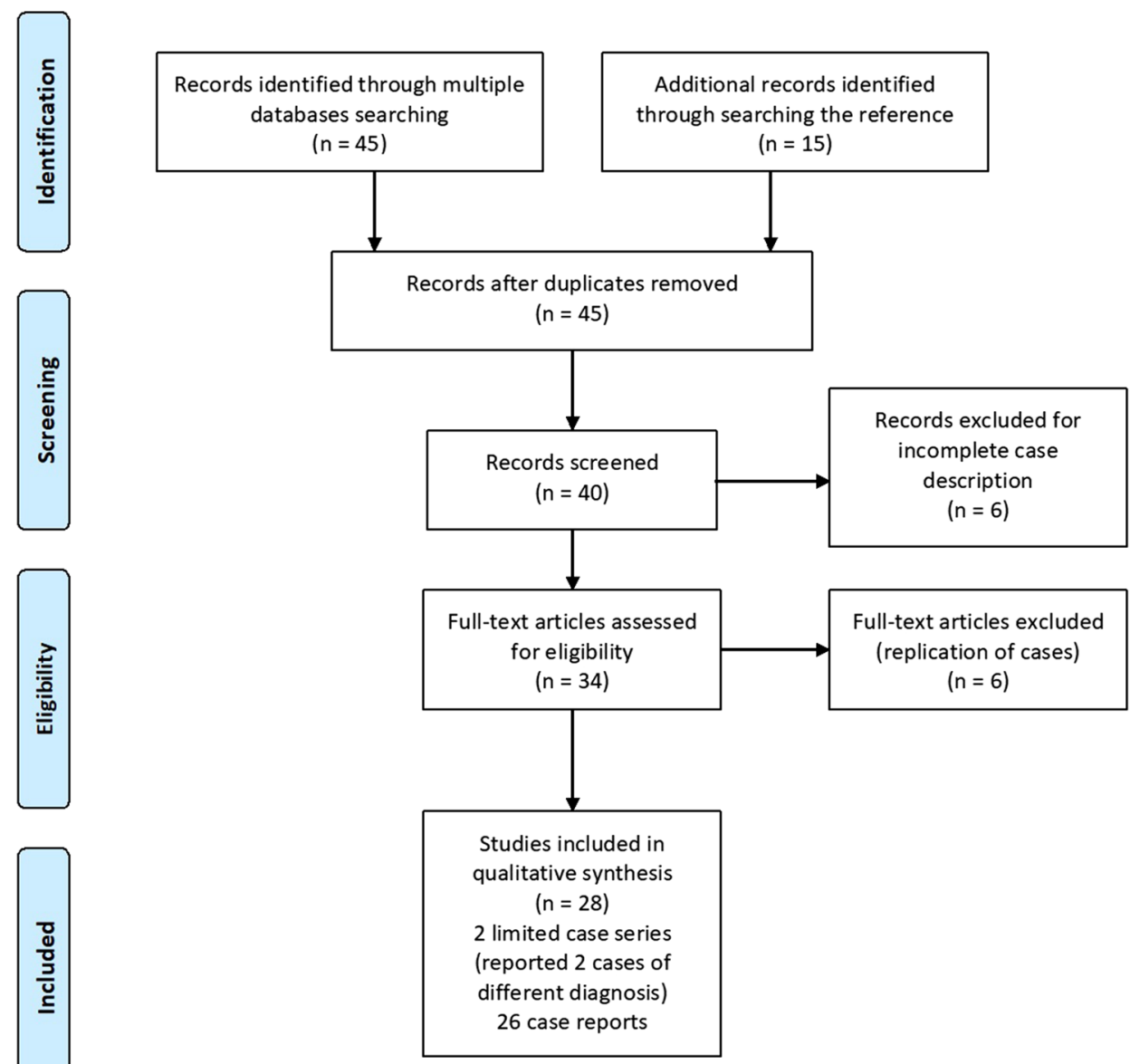

\section{Pathophysiological mechanism of autoimmune encephalitis in SARS-CoV-2 infection}

The exact pathophysiological mechanism of autoimmune encephalitis in SARS-CoV-2 infections is still yet to be understood. One of the most plausible proposed mechanisms was a molecular mimicry in response to the SARSCoV-2 infection which led to activation of host antibodies that cross-reacted and identified self antigens as foreign and cause damages to various system, including the central nervous system (CNS) [13]. There were various anti-neuronal autoantibodies that have been discovered to affect the nervous system in SARS-CoV-2 infected patients, namely the anti-N-methyl-D-aspartate-receptor (NMDAR) antibody [14-19], anti-contactin-associated protein-like-2 (CASPR2) antibodies [20], anti-myelin oligodendrocyte glycoprotein (MOG) antibody [21, 22], anti-glutamic acid decarboxylase (GAD) antibody [23], anti-GD1a antibody [24] and many others. This mechanism was also suggested to explain other neurological complications of
SARS-CoV-2 infection, namely Guillain-Barré syndrome [25] and acute disseminated encephalomyelitis [26], which further support the theory of molecular mimicry as the pathophysiology of autoimmune encephalitis.

Another proposed mechanism was systemic hyperinflammation due to over activation of host immune system causing the release of large amounts of inflammatory cytokines as a response to SARS-CoV-2 infection in a phenomenon known as "cytokine storm". These inflammatory mediators are transported throughout the body including the central nervous system and result in inflammatory damage or encephalitis [27]. This mechanism was further supported by the evidence from the laboratory finding on the serology and cerebral spinal fluid (CSF) analysis which shows elevated proinflammatory parameters such C-reactive protein and D-dimer $[11,28,29]$ and also the inflammatory mediators, such as the IL-6 [14, 16, 29]. In addition to that, the electroencephalogram (EEG) findings from some of the patients reveal diffuse patterns which are indicative of extensive inflammation [16, 19-21, 26, 30-32]. 


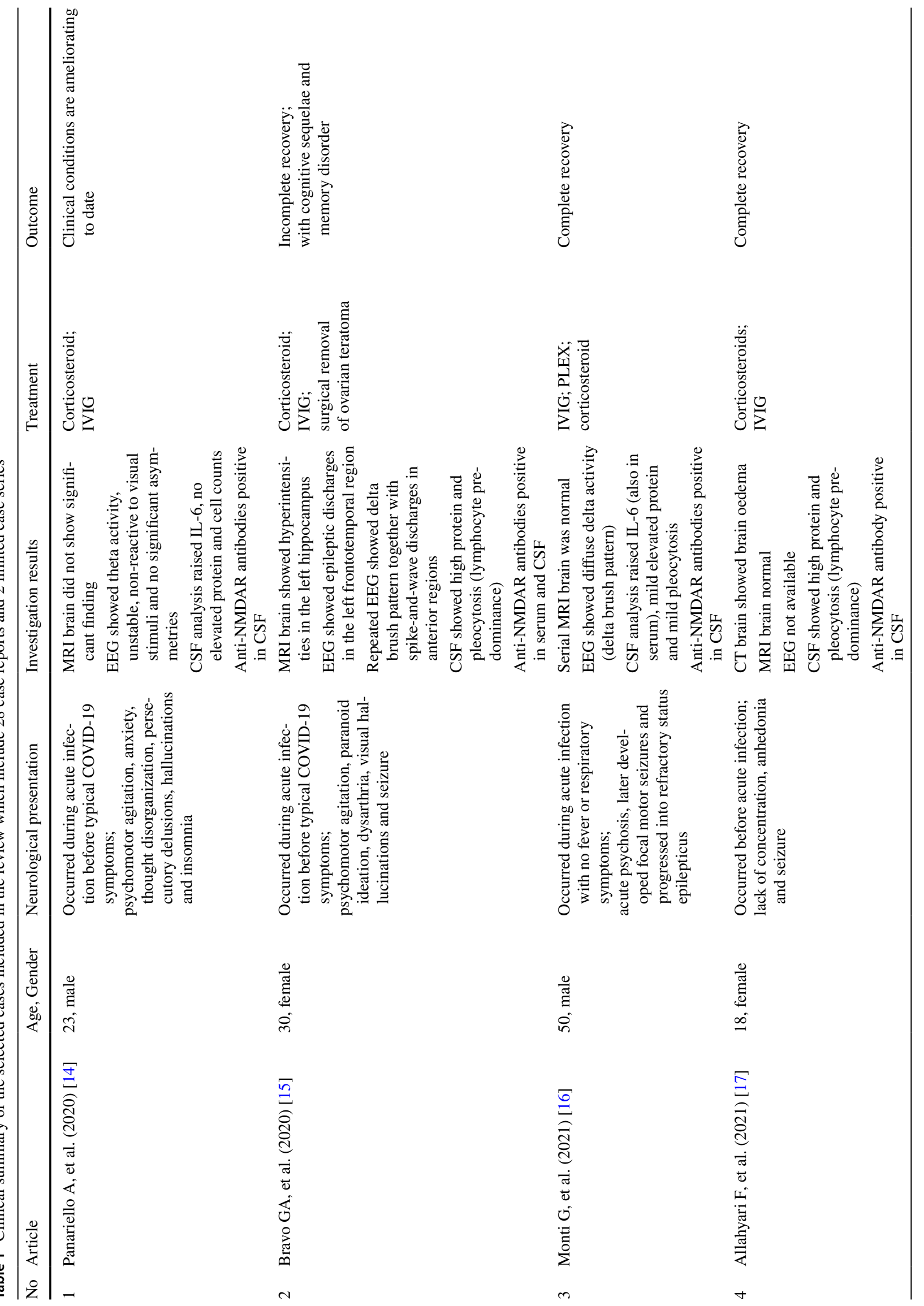




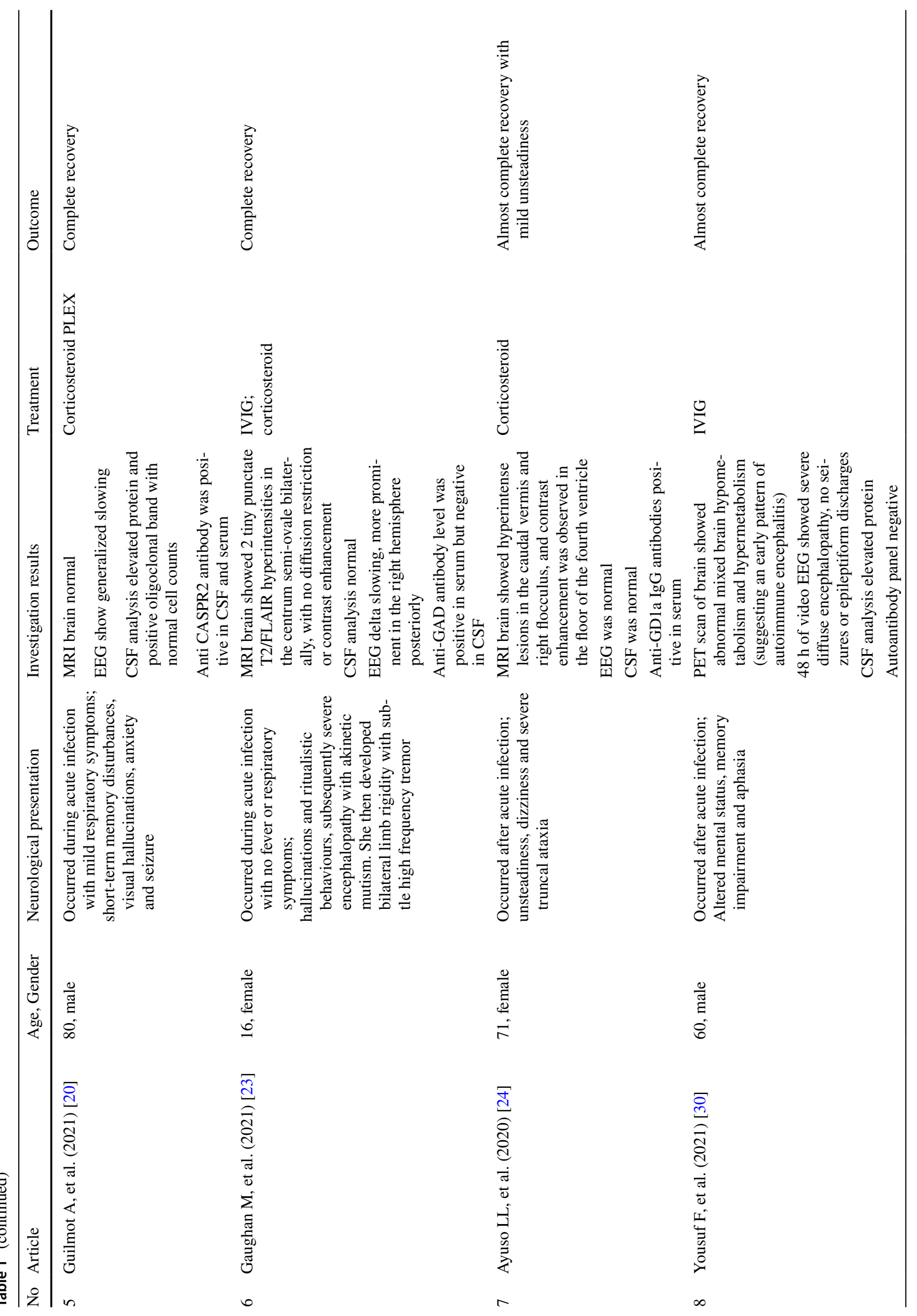




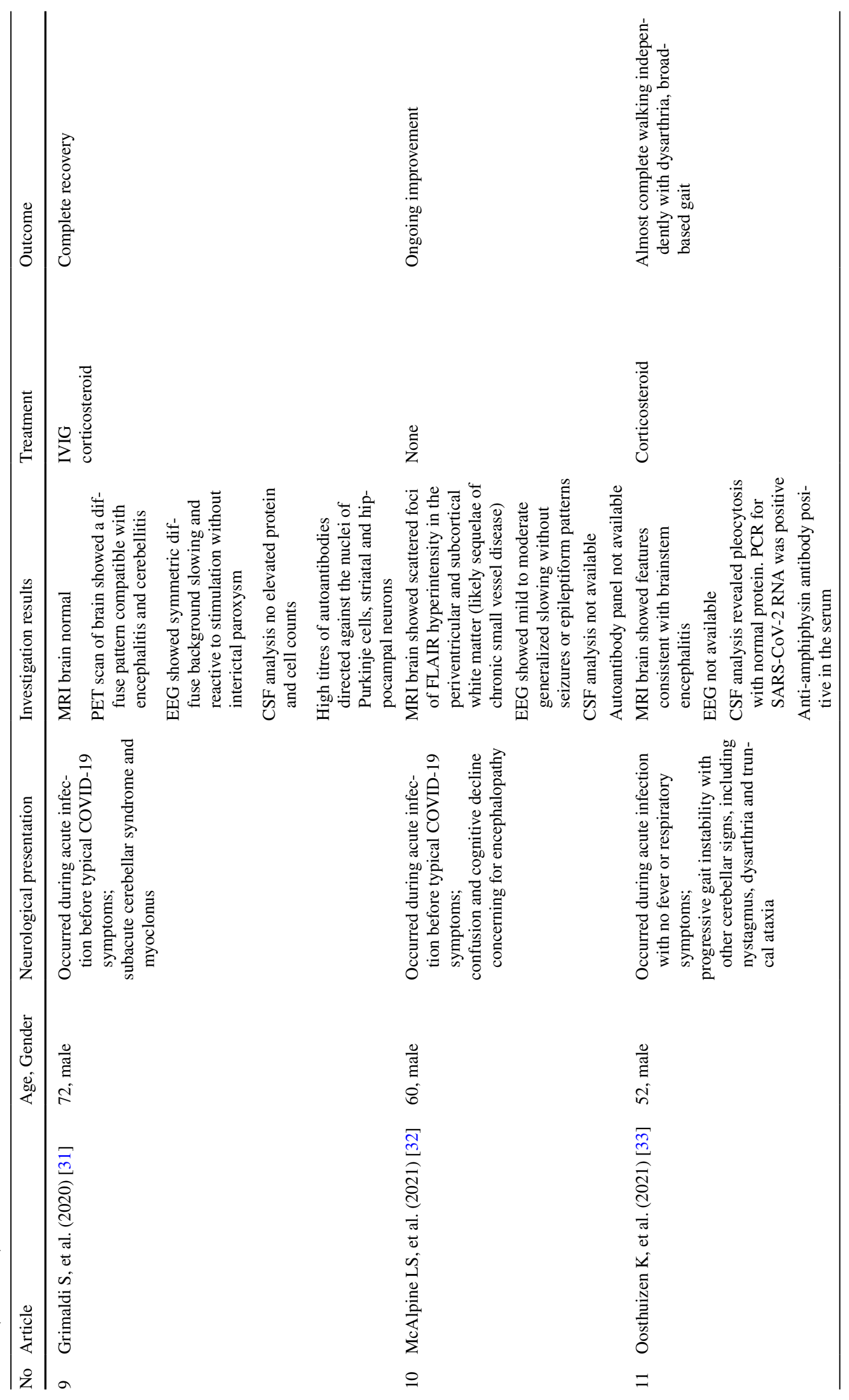




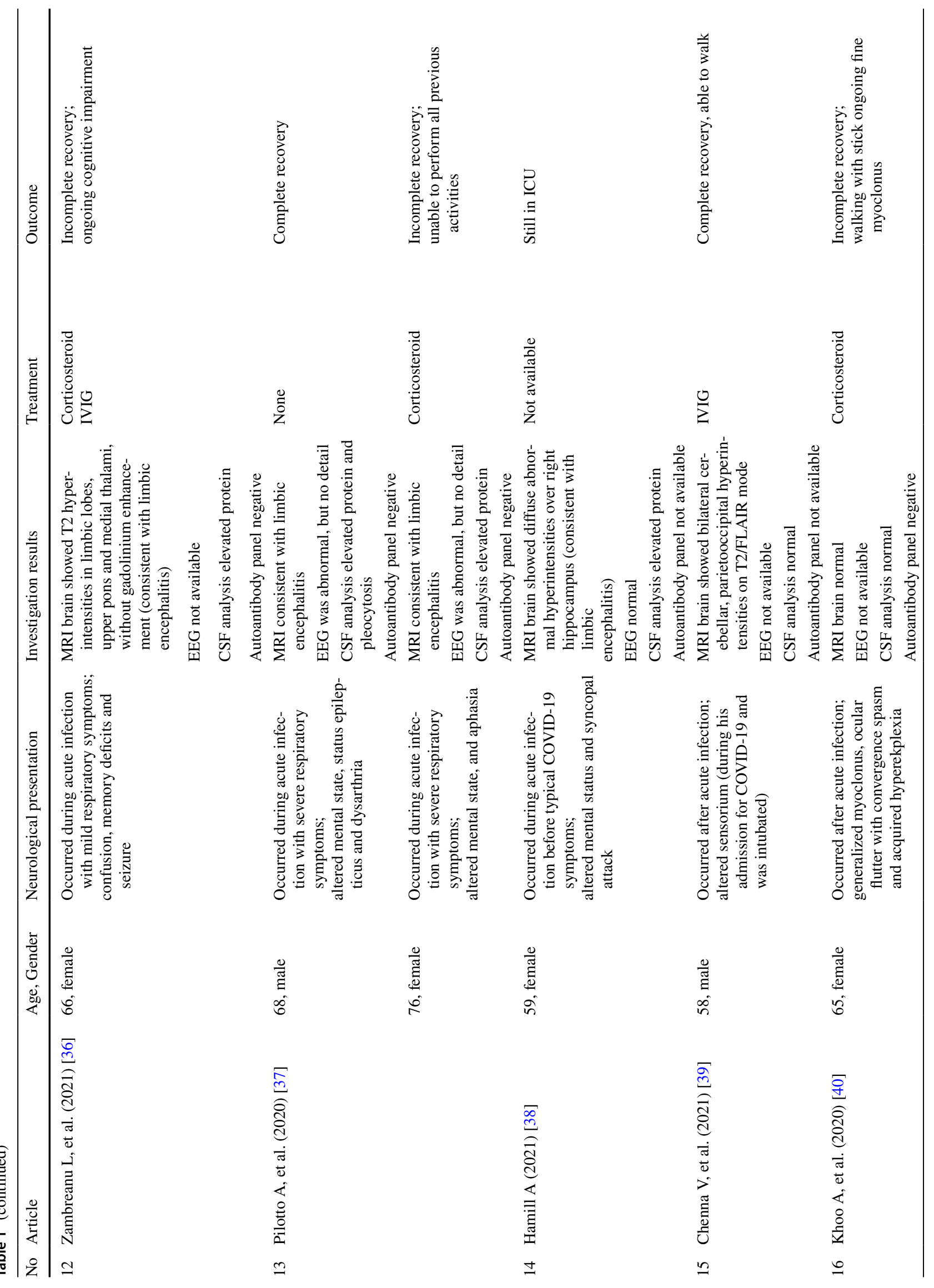




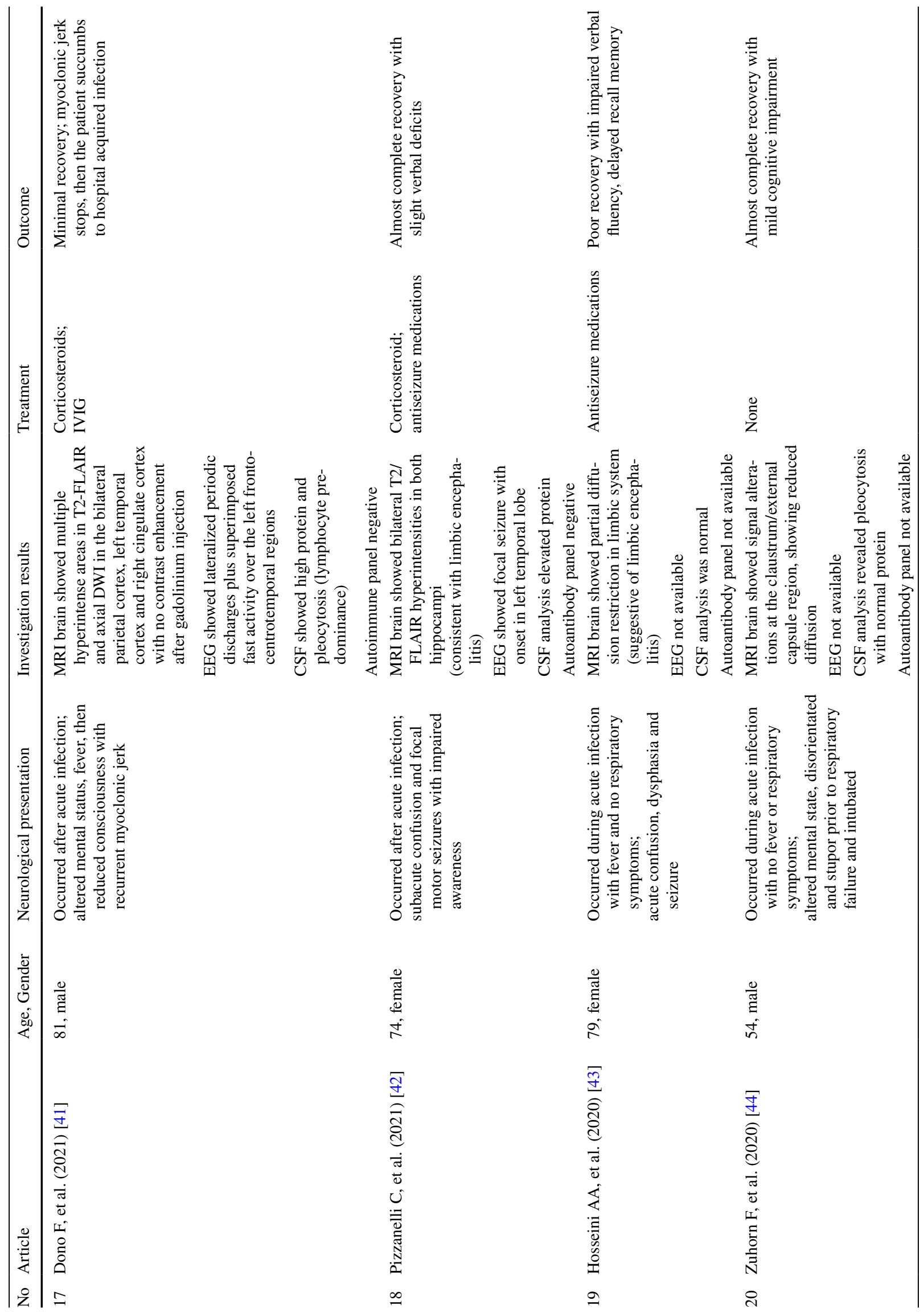




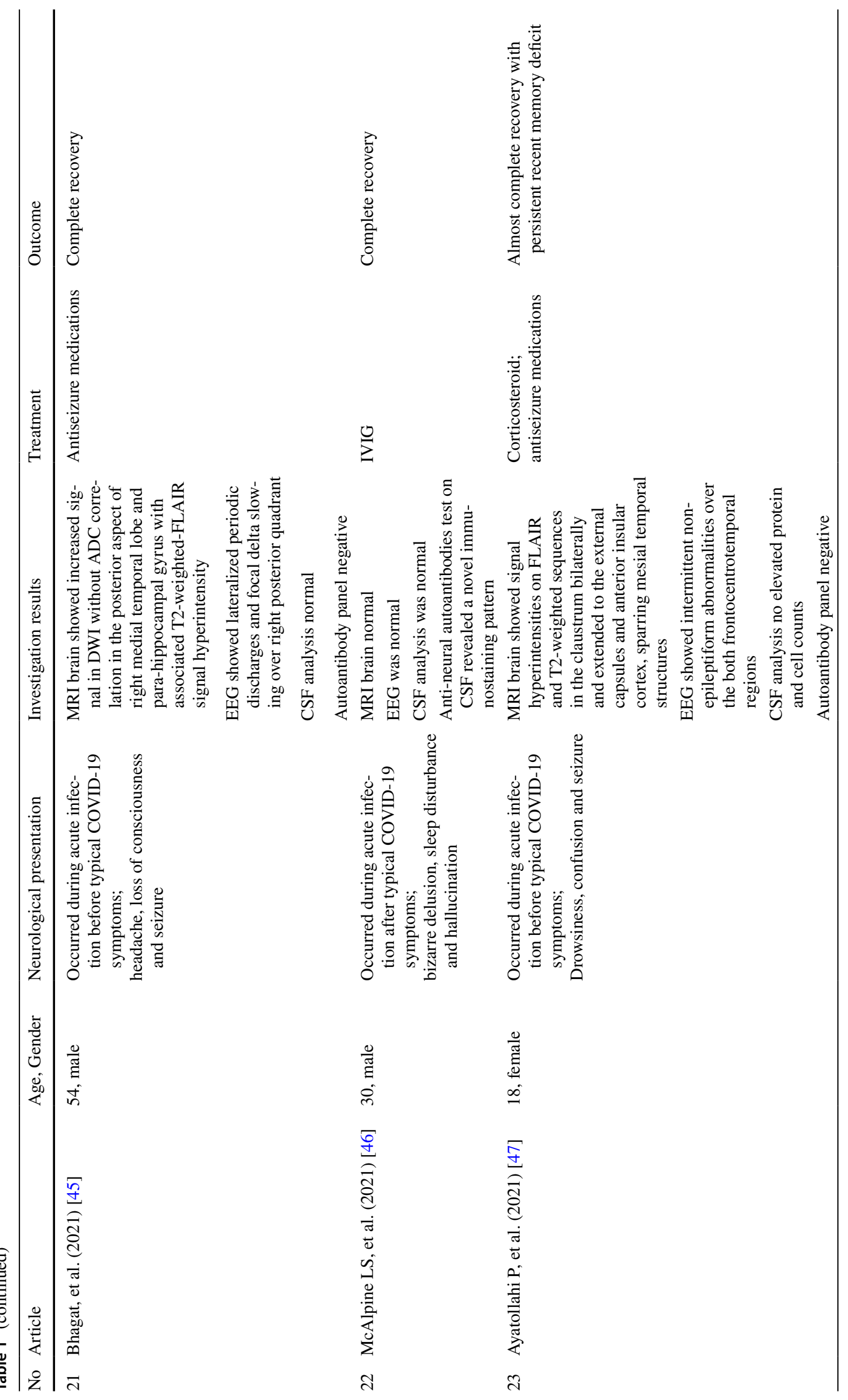




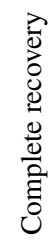

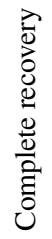

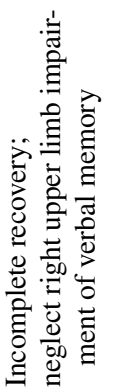

$\stackrel{0}{\geq}$

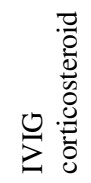

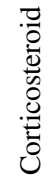

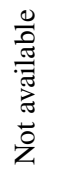

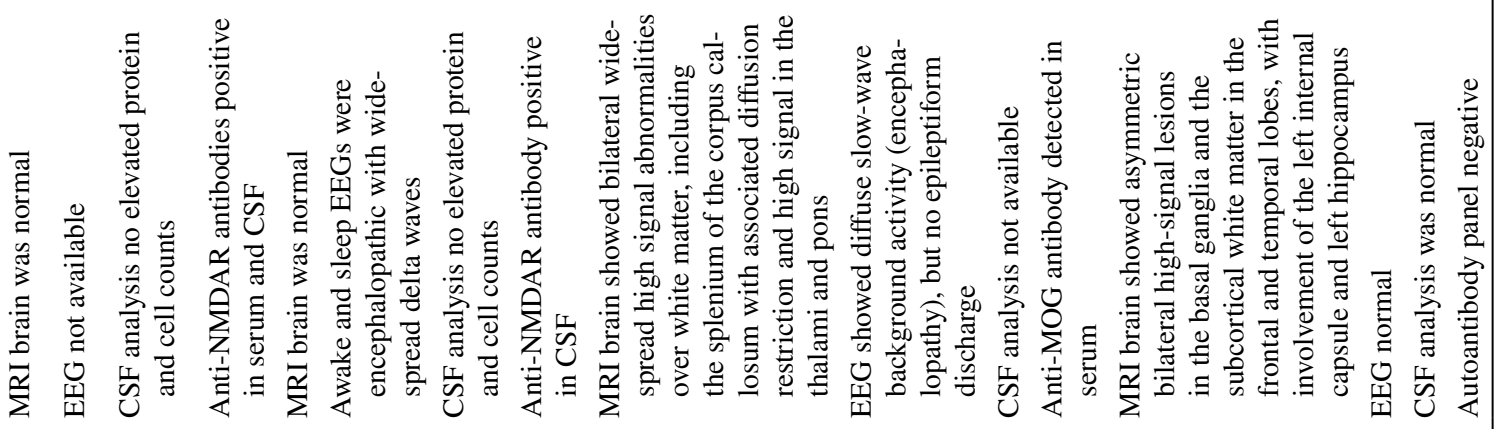

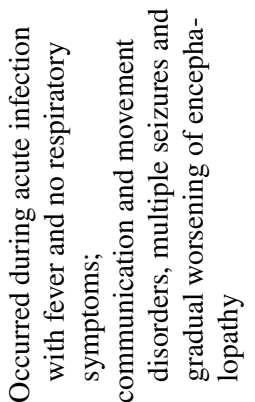
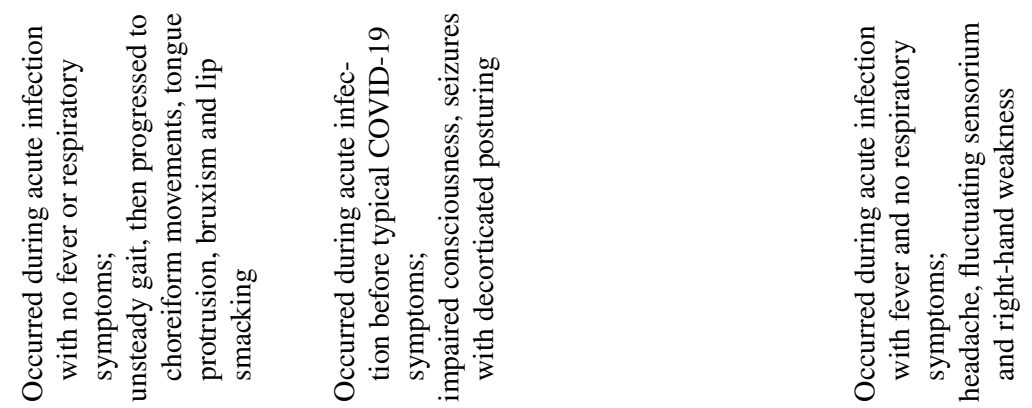

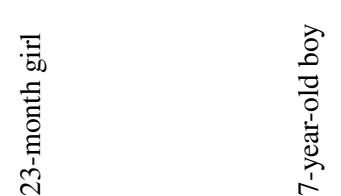

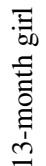

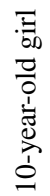

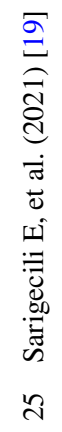

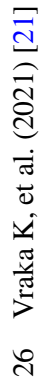

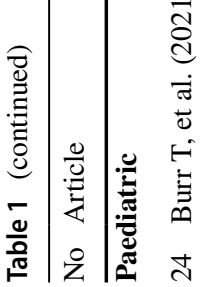




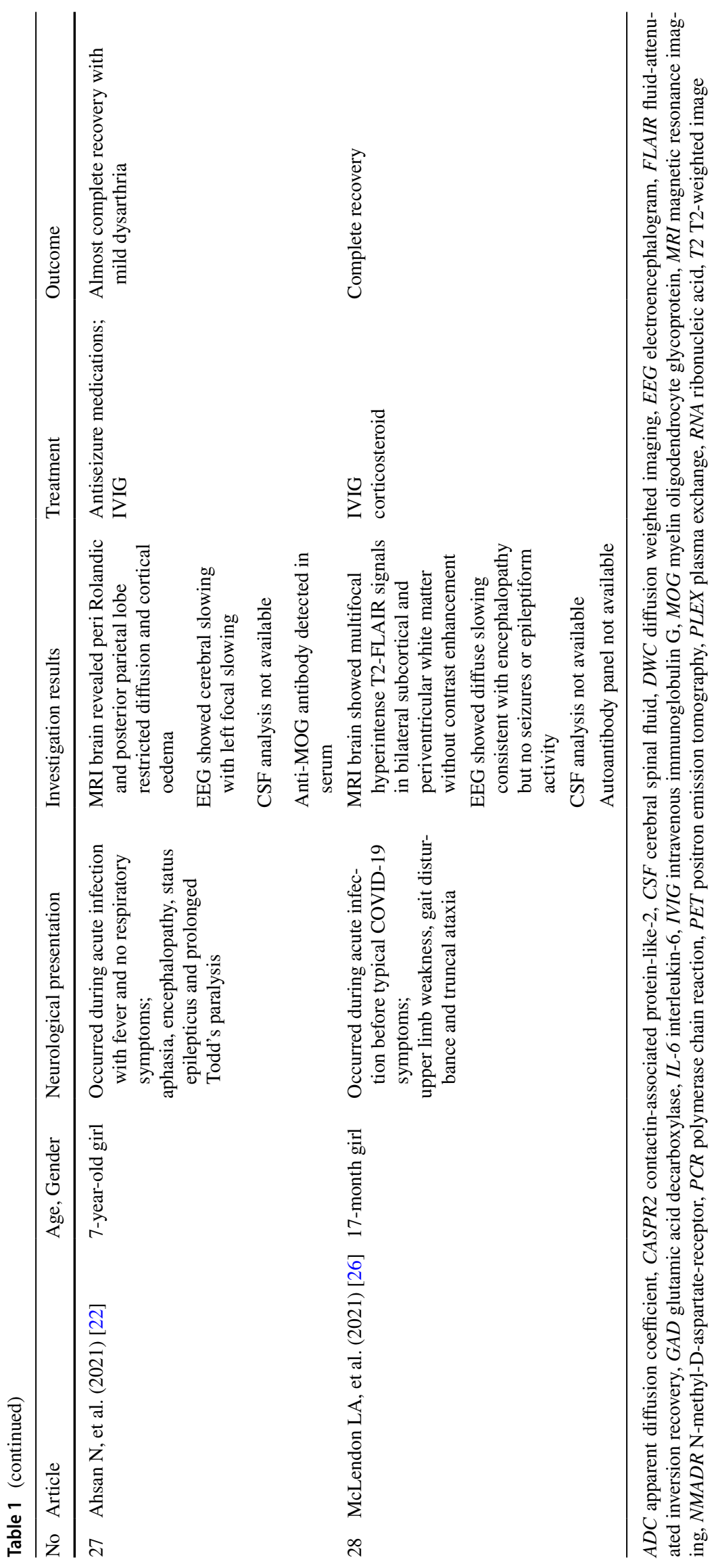


The third proposed pathophysiological mechanism was direct invasion of the virus into the central nervous system that led to the development of encephalitis. Since the earlier times of the pandemic, it has been postulated that the SARS$\mathrm{CoV}-2$ virus could enter the brain parenchyma through either the transsynaptic propagation or the haematogenous invasion [11]. Oosthuizen K, et al. [33] reported a case of middle-aged man with SARS-CoV-2 infection presented with progressive ataxia who was found to have SARS-CoV-2 particles detected via polymerase chain reaction (PCR) in the CSF. The patient was also noted to have positive antiamphiphysin antibody in the serum, which is an onconeural antibody that is usually associated with paraneoplastic encephalitis despite no malignancy found upon extensive full body screening. This may show that viral infections can trigger antibodies against cell surface antigens as it was well documented in the case of Herpes Simplex virus (HSV) encephalitis which can trigger anti-NMDAR encephalitis through uncovering epitopes resulting in antibody responses [34]. However, Siahaan YM, et al. [35] suggested that direct invasion of SARS-CoV-2 virus into the CNS is less likely to be the main mechanism of encephalitis in SARS-CoV-2 infection. This is because there are very limited cases where SARS-CoV-2 infected patients with encephalitis had positive PCR against SARS-CoV-2 detected in the CSF.

\section{Autoimmune encephalitis in SARS-CoV-2 infection}

From the cases that we reviewed in this article, $24(80 \%)$ of the patients were adults with the majority (58\%) of them aged more than 55 years old [20, 24, 30-32, 36-43]. Six (20\%) of the patients were from paediatric age, defined as 12 years and younger [18, 21-23, 26]. Amongst the most commonly reported neurological presentation of autoimmune encephalitis in SARS-CoV-2 infection are altered mental status [21, 30, 32, 36-39, 41-44, 47], psychomotor agitation $[14,15,20]$, memory deficit $[20,30,36]$, some form of psychogenic disturbance like hallucinations or delusions [14-16, 23, 46], ataxia [19, 24, 26, 31, 33] and seizure [15-18, 20-22, 36, 37, 42, 43, 45, 47]. Based on the typical findings on magnetic resonance imaging scan of the brain, six of the patients had limbic encephalitis [36-38, 42, 43], three had cerebellitis [24, 31, 39] and one had brain stem encephalitis [33]. There were 11 patients reported to develop autoantibodies either in the serum or/and in the cerebrospinal fluid (CSF) sample. Six of these autoantibodies were anti-NMDAR antibody [14-19], two were anti-MOG antibody [21, 22], and one anti-GAD [23], anti-GD1a [24] and anti-CASPR2 antibodies [20] each. Grimaldi S, et al. [31] reported a case of an elderly man who presented subacute cerebellar syndrome with myoclonus which occurs after the acute SARS-CoV-2 infection. His positron emission tomography scan of brain showed a diffuse pattern compatible with encephalitis and cerebellitis, and his CSF analysis revealed no elevated protein and normal cell counts, but there were high titres of autoantibodies directed against the nuclei of Purkinje cells, striatal and hippocampal neurons found. He was successfully treated with high-dose corticosteroid and intravenous (IV) immunoglobulin G and had complete recovery. McAlpine LS, et al. [32] on the other hand reported a novel immunostaining pattern from the antineural autoantibodies test on the CSF in a young male patient presented with bizarre delusion and hallucination. The patient was successfully treated with IV immunoglobulin $\mathrm{G}$ and achieved complete recovery.

In this review, majority (77\%) of the patients had the autoimmune encephalitis occurred during or concurrent with the acute infection of the SARS-CoV-2 which is termed "para-infectious presentation" [14-16, 18-23, 26, 31-33, $36-39,44-47]$. From this, $30 \%$ of the patients presented with neurological manifestations before developing fever and respiratory symptoms $[14,15,21,26,31,32,38,45$, 47]. Six (20\%) of the cases occurred after the acute infection [24, 30, 40-43], and only one (3\%) occurred before the acute infection which was reported by Allahyari F, et al. [17] in a young female with anti-NMDAR antibody positive in CSF. She was successfully treated with high-dose corticosteroid and IV immunoglobulin $\mathrm{G}$ with complete recovery.

There were 18 patients that were treated with high-dose corticosteroid, which is either oral prednisolone $(1 \mathrm{mg} / \mathrm{kg}$, max $60 \mathrm{mg}$ ) daily or pulsed intravenous (IV) methylprednisolone $1 \mathrm{~g}$ daily [14-17, 19-21, 23, 24, 26, 31, 33, 36, $37,40-42,47]$. On the other hand, 15 patients were treated with IV immunoglobulin G [14-19, 22, 23, 26, 30, 31, 36, $39,41,46]$. There were two patients that were treated with just antiseizure medication [43, 45], and three patients were not given any treatment at all $[32,37,44]$. There were nine patients that received a combination of high-dose corticosteroid and IV immunoglobulin G [14, 15, 17, 19, 23, 26, 31, $36,41]$, and two were subjected into plasma exchange [16, 20]. Monti $\mathrm{G}$ et al. [16] reported a young patient with antiNMDAR antibodies positive in the CSF which was treated with a combination of plasma exchange, IV immunoglobulin $\mathrm{G}$ and corticosteroid and had complete recovery. Guilmot A., et al. [20] on the other hand reported an elderly patient with positive anti-CASPR2 antibody in both CSF and serum which was successfully treated with high-dose corticosteroid and plasma exchange and had complete recovery.

The prognosis of autoimmune encephalitis in SARSCoV-2 infection based on all the cases in our study was generally good where $20(67 \%)$ of the patients have complete or almost complete recovery [16-24, 26, 30, 31, 33, $37,39,42-47]$. There were five $(17 \%)$ patients that have incomplete recovery $[15,21,36,37,40]$, and only two (7\%) patients had poor recovery, which was the case of limbic 
encephalitis reported by Hosseini AA, et al. [43] and a case of post-SARS-CoV-2 infection autoimmune encephalitis presenting as new-onset refractory status epilepticus which was reported by Dono F, et al. [41].

\section{Autoimmune encephalitis in SARS-CoV-2 infection in paediatric}

Out of the 30 patients that were reviewed in this article, there were six paediatric patients defined as age 12 years and younger. Vraka K, et al. [21] reported a case of an anti-MOG antibody positive in the serum of a 13-month-old girl. The young girl was successfully treated with high-dose corticosteroid and achieved complete recovery. Another similar case was reported in a 7-year-old girl by Ahsan N, et al. [22] who also had anti-MOG antibody positive in the serum. She was treated with IV immunoglobulin $\mathrm{G}$ and had a good recovery with only mild dysarthria. Burr T, et al. [18] and Sarigecili E, et al. [19] on the other hand reported a 23-month-old girl and a 7-year-old boy with anti-NMDAR IgG antibody which were both treated with a combination of high-dose corticosteroid and IV immunoglobulin $\mathrm{G}$ and had a complete and almost complete recovery. It is worth to mention that not all of the paediatric patients have autoantibody positive in the serum or CSF. Vraka K, et al. [21] reported another case in their article on a 10-year-old girl who presented with righthand weakness and fluctuating sensorium. She has normal CSF analysis findings and negative autoantibody panel. She was not given any immunomodulating therapy and still has neglect over her right hand and impairment of her verbal memory. Another case was reported by McLendon LA, et al. [26] in a 17-month-old girl who developed upper limb weakness and ataxia, which was also treated with a combination of high-dose corticosteroid and IV immunoglobulin $\mathrm{G}$ and had complete and almost complete recovery. However, the authors did not include the CSF analysis and the autoantibody panel test results in the report.

\section{Discussion}

SARS-CoV-2 belongs to the genus of beta-coronavirus which is a zoonotic disease that can infect both humans and animals. Since it was first reported in December 2019, the infection has spread rapidly across country borders and caused massive global pandemic. Up to date, there are more than 200 million reported confirmed cases of COVID-19, and more than four million deaths have taken place from more than 150 countries around the world [48]. Infection by SARS-CoV-2 causes a typical clinical syndrome such as fever, cough, shortness of breath, myalgia and fatigue. Other clinical presentations that are considered non-typical are diarrhoea, anorexia, conjunctival congestion, nausea and vomiting. About 20 to $30 \%$ of patients may progress to more critical conditions with acute respiratory distress syndrome, septic shock, disseminated intravascular coagulation, acute heart failure and acute kidney injury [49]. Another peculiarity about SARS-CoV-2 infection is the propensity to affect the nervous system. In our previous studies, we reviewed multiple clinical evidences that show SARS-CoV-2 involvement on the nervous system and raised a major concern on the possibility of future autoimmune complication involving the nervous system [11]. There have been multiple cases that have been reported on the possible autoimmune occurrence in SARS-CoV-2 infection. Therefore, here we studied the link between autoimmune encephalitis in SARS-CoV-2 infection and the prognosis of the condition.

Similar to what have been observed in autoimmune disease involving the nervous system induced by other viral infections like HSV which itself is more common to cause encephalitis [34], autoimmune encephalitis can occur after the resolution or more commonly during the acute phase of SARS-CoV-2 infection. It can happen in any spectrum of disease severity, and some can even occur without any of the typical symptoms of COVID-19. Apart from that, there were various types of serum and CSF autoantibodies related to encephalitis that have been reported, namely antigangliosides antibody (such as anti-GAD antibody [23] and anti-GD1a antibody [24]), anti-neuronal antibodies (antiNMDAR antibody [14-19] and anti-CASPR2 antibody [20]) and onconeural antibodies (anti-amphiphysin antibody [33]). The clinical significance of the anti-amphiphysin antibodies in the case reported by Oosthuizen K, et al. [33] was unclear given that no malignancy was identified in the patient. However, the RNA of SARS-CoV-2 was detected in the CSF sample of the patient, which could indicate direct invasion of the virus to the CNS causing encephalitis that may have induced responses against amphiphysin, which is an intracellular synaptic antigen. However, the association still warrants future studies for further explanation and to explore the associations between SARS-CoV-2 infection and immunemediated CNS disease.

The possibility of immune-mediated neurology disease in SARS-CoV-2 infection leading to future disabilities is still a major concern. This is because the unknown complications can occur years after the recovery from acute infection, especially when evidence have shown other types of coronaviruses that are linked to central nervous system dysfunction such as multiple sclerosis [50]. Furthermore, there were cases where antibody against MOG, which is a myelin protein that exclusively expressed on the surface of CNS myelin sheaths, was reported amongst the paediatric patients $[21,22]$. Antibody against MOG has been associated with various autoimmune inflammatory demyelinating diseases, such as multiple sclerosis and acute disseminated encephalomyelitis (ADEM). ADEM is an autoimmune 
demyelinating disease that commonly affects the brainstem and spinal cord and typically presents as a monophasic disorder associated with multifocal neurologic symptoms and encephalopathy [22]. Therefore, more reporting of cases and studies are deemed necessary to illuminate the link and potential reversibility of the neurological syndrome, and the overall impact of the infection in promoting other neurological diseases in the future.

\section{Limitations of the study}

This study has limitations, where most of the articles analysed are case reports and limited case series. Apart from that, this article mainly uses descriptive analysis to review and summarize the clinical cases.

\section{Conclusion}

In conclusion, primary data on COVID-19 patients with autoimmune encephalitis remains limited. Therefore, this review aims to illuminate the occurrence of autoimmune encephalitis in SARS-CoV-2 infection based on the evidence presented from the previous published case reporting and also attempt to educate clinicians around the world regarding the conditions in order to improve the prognosis of COVID19 by delivering the appropriate treatment promptly.

Acknowledgements The authors would like to thank to all who were directly and indirectly involved in the writing and publishing of this review article.

\section{Declarations}

Ethics approval and consent to participate None.

Conflict of interest The authors declare no competing interests.

\section{References}

1. Zhu N, Zhang D, Wang W, Li X, Yang B, Song J et al (2020) A novel coronavirus from patients with pneumonia in China. $\mathrm{N}$ Engl J Med 2019. https://doi.org/10.1056/NEJMoa2001017

2. Li Q, Guan X, Wu P, Wang X, Zhou L, Tong Y et al (2020) Early transmission dynamics in Wuhan, China, of novel coronavirusinfected pneumonia. N Engl J Med. https://doi.org/10.1056/ NEJMoa2001316

3. Schoeman D, Fielding BC (2019) Coronavirus envelope protein: current knowledge. Virol J 16(1):69

4. Gorbalenya AE, Baker SC, Baric R, Groot RJ, Drosten C, Gulyaeva AA, Haagmans BL, Lauber C, Leontovich AM, Neuman BW, Penzar D. Severe acute respiratory syndrome-related coronavirus: the species and its viruses - a statement of the Coronavirus Study Group
5. Chen Y, Liu Q, Guo D (2020) Emerging coronaviruses: genome structure, replication, and pathogenesis. J Med Virol 92(4):418-423

6. Su S, Wong G, Shi W, Liu J, Lai ACK, Zhou J et al (2016) Epidemiology, genetic recombination, and pathogenesis of coronaviruses. Trend Microbiol 24:490-502

7. Peiris JS, Guan Y, Yuen K (2004) Severe acute respiratory syndrome. Nature Med 10:S88-97

8. Chan-Yeung M, Xu RH. SARS: epidemiology. Respirology 8 Suppl S9-14

9. Zaki AM, Boheemen SV, Bestebroer TM, Osterhaus ADME, Fouchier RAM (2012) Isolation of a novel coronavirus from a man with pneumonia in Saudi Arabia. N Engl J Med 367:1814-1820

10. Huang C, Wang Y, Li X, Ren L, Zhao J, Hu Y et al (2020) Clinical features of patients infected with 2019 novel coronavirus in Wuhan, China. Lancet 395:497-506

11. Payus AO, Lin CL, Noh MM, Jeffree MS, Ali RA (2020) SARSCoV-2 infection of the nervous system: a review of the literature on neurological involvement in novel coronavirus disease(COVID-19). Bosn J Basic Med Sci 20(3):283

12. Toussirot É, Roudier J (2008) Epstein-Barr virus in autoimmune diseases. Best Pract Res Clin Rheumatol 22(5):883-896

13. Siow I, Lee KS, Zhang JJ, Saffari SE, Ng A (2021) Encephalitis as neurological complication of COVID-19: a systematic review and meta analysis of incidence, outcomes and predictors. Eur J Neurol

14. Panariello A, Bassetti R, Radice A, Rossotti R, Puoti M, Corradin M, Moreno M, Percudani M (2020) Anti-NMDA receptor encephalitis in a psychiatric Covid-19 patient: a case report. Brain Behav Immun 87:179

15. Bravo GA, Torrentà LR (2020) Anti-NMDA receptor encephalitis secondary to SARS-CoV-2 infection. Neurología (Engl Ed) 35(9):699

16. Monti G, Giovannini G, Marudi A, Bedin R, Melegari A, Simone AM, Santangelo M, Pignatti A, Bertellini E, Trenti T, Meletti S (2020) Anti-NMDA receptor encephalitis presenting as new onset refractory status epilepticus in COVID-19. Seizure 81:18

17. Allahyari F, Hosseinzadeh R, Nejad JH, Heiat M, Ranjbar R (2021) A case report of simultaneous autoimmune and COVID19 encephalitis. J Neurovirol 1-3

18. Burr T, Barton C, Doll E, Lakhotia A, Sweeney M (2021) $\mathrm{N}$-methyl-d-aspartate receptor encephalitis associated with COVID-19 infection in a toddler. Pediatr Neurol 114:75

19. Sarigecili E, Arslan I, Ucar HK, Celik U (2021) Pediatric antiNMDA receptor encephalitis associated with COVID-19. Child's Nervous Syst 1-4

20. Guilmot A, Slootjes SM, Sellimi A, Bronchain M, Hanseeuw B, Belkhir L, Yombi JC, De Greef J, Pothen L, Yildiz H, Duprez T (2021) Immune-mediated neurological syndromes in SARS-CoV2-infected patients. J Neurol 268(3):751-757

21. Vraka K, Ram D, West S, Chia WY, Kurup P, Subramanian G, Tan HJ (2021) Two paediatric patients with encephalopathy and concurrent COVID-19 infection: two sides of the same coin? Case Rep Neurol Med 2021

22. Ahsan N, Jafarpour S, Santoro JD (2021) Myelin oligodendrocyte glycoprotein antibody encephalitis following severe acute respiratory syndrome coronavirus 2 in a pediatric patient. Clin Exp Pediatr 64(6):310

23. Gaughan M, Connolly S, O'Riordan S, Tubridy N, McGuigan C, Kinsella JA (2021) Pediatric parainfectious encephalitis associated with COVID-19. Neurology 96(11):541-544

24. Ayuso LL, Rubio PT, do Rosário RF, Arroyo ML, Sierra-Hidalgo F (2020) Bickerstaff encephalitis after COVID-19. J Neurol 1-3

25. Payus AO, Jan T, Ibrahim A, Raymond AA (2020) Autoimmune polyradiculopathy In Sars-Cov-2: a narrative review of 
Guillain-Barre syndrome in novel coronavirus disease (Covid19). Acta Med 36:3781

26. McLendon LA, Rao CK, Da Hora CC, Islamovic F, Galan FN (2021) Post-COVID-19 acute disseminated encephalomyelitis in a 17-month-old. Pediatrics 147(6)

27. Scoppettuolo P, Borrelli S, Naeije G (2020) Neurological involvement in SARS-CoV-2 infection: a clinical systematic review. Brain Behav Immun Health. 5:100094

28. Paterson RW, Brown RL, Benjamin L, Nortley R, Wiethoff S, Bharucha T, Jayaseelan DL, Kumar G, Raftopoulos RE, Zambreanu L, Vivekanandam V (2020) The emerging spectrum of COVID-19 neurology: clinical, radiological and laboratory findings. Brain 143(10):3104-3120

29. Hambali NL, Noh MM, Paramasivam S, Chua TH, Hayati F, Payus AO, Tee TY, Rosli KT, Isnadi MF, Manin BO (2020) A non-severe coronavirus disease 2019 patient with persistently high interleukin-6 level. Front Public Health 8

30. Yousuf F, King RD, Ainger T, Yamasaki T, Wilcock DM, Lee $\mathrm{TL}$, Hessler AB. Autoimmune encephalitis following recovery of acute SARS-CoV-2 infection severe acute respiratory syndrome coronavirus 2 infection

31. Grimaldi S, Lagarde S, Harlé JR, Boucraut J, Guedj E (2020) Autoimmune encephalitis concomitant with SARS-CoV-2 infection: insight from 18F-FDG PET imaging and neuronal autoantibodies. J Nucl Med 61(12):1726-1729

32. McAlpine LS, Barden M, Zubair AS, Sanamandra S (2021) Isolated encephalopathy without severe disease in a COVID-19 patient: case presentation and workup strategies. Cureus 13(2)

33. Oosthuizen K, Steyn EC, Tucker L, Ncube IV, Hardie D, Marais S (2021) SARS-CoV-2 encephalitis presenting as a clinical cerebellar syndrome: a case report. Neurology 97(1):27-29

34. Armangue T, Spatola M, Vlagea A, Mattozzi S, Cárceles-Cordon M, Martinez-Heras E, Llufriu S, Muchart J, Erro ME, Abraira L, Moris G (2018) Frequency, symptoms, risk factors, and outcomes of autoimmune encephalitis after herpes simplex encephalitis: a prospective observational study and retrospective analysis. Lancet Neurol 17(9):760-772

35. Siahaan YM, Puspitasari V, Pangestu AR. COVID-19-associated encephalitis: systematic review of case reports findings on cytokine-immune-mediated inflammation as an underlying mechanism.

36. Zambreanu L, Lightbody S, Bhandari M, Hoskote C, Kandil H, Houlihan CF, Lunn MP (2020) A case of limbic encephalitis associated with asymptomatic COVID-19 infection. J Neurol Neurosurg Psychiatry 91(11):1229-1230

37. Pilotto A, Masciocchi S, Volonghi I, del Zotto E, Magni E, De Giuli V, Caprioli F, Rifino N, Sessa M, Gennuso M, Cotelli MS (2020) The clinical spectrum of encephalitis in COVID-19 disease: the ENCOVID multicentre study. Medrxiv
38. Hamill A (2021) Limbic encephalitis as a consequence of COVID19 infection. Available at SSRN 3773159

39. Chenna V, Jenjeti C, Devarapu K (2021) Use of intravenous immunoglobulin to successfully treat COVID-19 associated encephalitis. Neuroimmunol Neuroinflam 8

40. Khoo A, McLoughlin B, Cheema S, Weil RS, Lambert C, Manji H, Zandi MS, Morrow JM (2020) Postinfectious brainstem encephalitis associated with SARS-CoV-2. J Neurol Neurosurg Psychiatry 91(9):1013-1014

41. Dono F, Carrarini C, Russo M, De Angelis MV, Anzellotti F, Onofrj M, Bonanni L (2021) New-onset refractory status epilepticus (NORSE) in post SARS-CoV-2 autoimmune encephalitis: a case report. Neurol Sci 42(1):35-38

42. Pizzanelli C, Milano C, Canovetti S, Tagliaferri E, Turco F, Verdenelli S, Nesti L, Franchi M, Bonanni E, Menichetti F, Volterrani D (2021) Autoimmune limbic encephalitis related to SARSCoV-2 infection: case-report and review of the literature. Brain Behav Immun Health 100210

43. Hosseini AA, Shetty AK, Sprigg N, Auer DP, Constantinescu CS (2020) Delirium as a presenting feature in COVID-19: neuroinvasive infection or autoimmune encephalopathy? Brain Behav Immun 88:68

44. Zuhorn F, Omaimen H, Ruprecht B, Stellbrink C, Rauch M, Rogalewski A, Klingebiel R, Schäbitz WR (2020) Parainfectious encephalitis in COVID-19: "The Claustrum Sign". J Neurol 1-4

45. Bhagat R, Kwiecinska B, Smith N, Peters M, Shafer C, Palade A, Sagi V (2021) New-onset seizure with possible limbic encephalitis in a patient with COVID-19 infection: a case report and review. J Investig Med High Impact Case Rep 9:2324709620986302

46. McAlpine LS, Lifland B, Check JR, Angarita GA, Ngo TT, Pleasure SJ, Wilson MR, Spudich SS, Farhadian SF, Bartley CM (2021) Remission of subacute psychosis in a COVID-19 patient with an antineuronal autoantibody after treatment with intravenous immunoglobulin. Biol Psychiatry

47. Ayatollahi P, Tarazi A, Wennberg R (2021) Possible autoimmune encephalitis with claustrum sign in case of acute SARS-CoV-2 infection. Can J Neurol Sci 48(3):430-432

48. World Health Organization. Situation reports. Available at: https:// www.who.int/emergencies/diseases/novel-coronavirus-2019/situa tion-reports/

49. Singhal T (2020) A review of coronavirus disease-2019 (COVID19). Indian J Pediatr 1-6

50. Boucher A, Desforges M, Duquette P, Talbot PJ (2007) Long-term human coronavirus-myelin cross-reactive T-cell clones derived from multiple sclerosis patients. Clin Immunol 123(3):258-267

Publisher's note Springer Nature remains neutral with regard to jurisdictional claims in published maps and institutional affiliations. 